\title{
Hypertension research: perspectives and progress. A message from the new Editor-in-Chief
}

\author{
Kazuomi Kario ${ }^{1}$
}

Published online: 5 January 2021

(C) The Japanese Society of Hypertension 2021

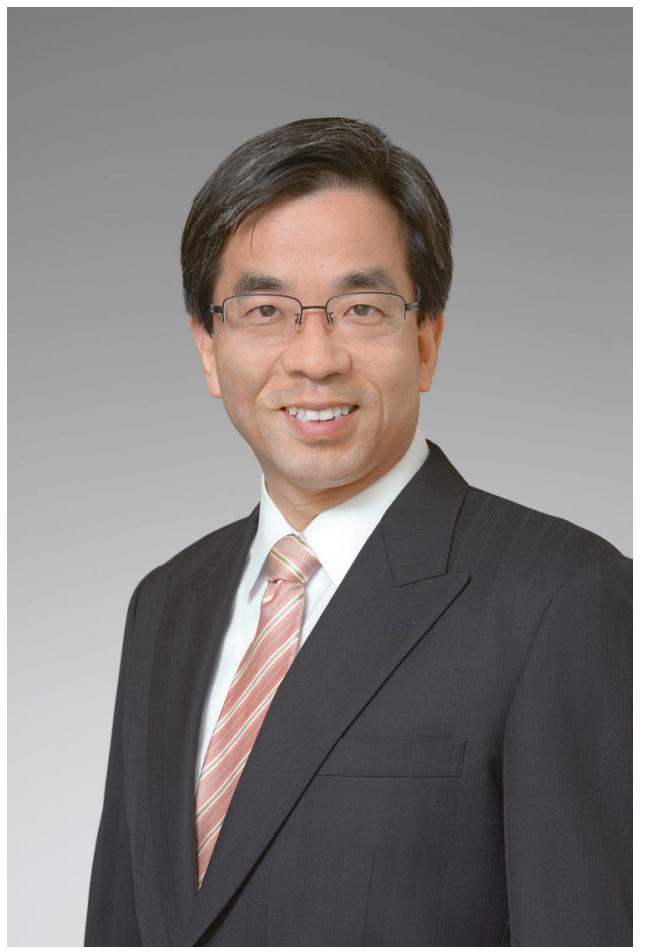

It is a great honor to introduce myself as the new, tenth Editor-in-Chief of Hypertension Research. The role of Editor-in-Chief is a significant responsibility, and I would like to thank the Japanese Society of Hypertension for the trust they have placed in me to lead the journal.

The first issue of the Japanese Journal of Hypertension, the official journal of the Japanese Society of Hypertension, was published in April 1978. Fourteen years later, in

Kazuomi Kario

kkario@jichi.ac.jp

1 Division of Cardiovascular Medicine, Department of Medicine, Jichi Medical University School of Medicine,

Shimotsuke, Tochigi, Japan
April 1992, the journal was revised and renamed as Hypertension Research, the official English language journal of the Japanese Society of Hypertension. A series of expert editors have guided the journal since then, including the first Editor-in-Chief, Tatsuro Kokubu (1992-1995), followed by Osamu IImura (1995-1999), Kunio Hiwada (1999-2002), Toshiro Fujita (2002-2004), Kazuyuki Shimada (2004-2008), Masatsugu Horiuchi (2009-2013), Shokei Kim-Mitsuyama (2013), Ryuichi Morishita (2013) and Toshihiko Ishimitsu (2014-2020). In particular, the longest and most recent 7-year period of leadership by Professor Toshihiko Ishimitsu has seen the publication of high-quality original papers and interesting up-to-date review papers written by leading researchers from around the world. The journals activities have been facilitated by Springer Nature since 2009, allowing dissemination of a range of academic activities in a journal that ranks highly in its field. I am passionate about the field of hypertension and cardiovascular disease prevention, and am committed to help continue the growth of Hypertension Research over the coming years. We can build on the excellent work of my predecessors to further expand the academic and scientific significance of the journal.

Hypertension Research will continue to publish valuable original research papers, as well as high-quality up-to-date reviews to provide guidance and commentary from international experts in the field. International collaboration is essential for the building and transfer of knowledge, and I am committed to facilitating relationships and interactions with societies such as the World Hypertension League, International Society of Hypertension, European Society of Hypertension and the American Heart Association to ensure that the messaging around hypertension is robust and consistent. In addition, academic research activity in the field of hypertension can be facilitated by interaction with other international journals in the field, such as Hypertension, Journal of Hypertension, Journal of Clinical Hypertension, Journal of Human Hypertension, and American Journal of Hypertension. Together we can play an integral role in the 
dissemination of international hypertension research, including basic science, epidemiology, case reports with review list and clinical studies. Other important tasks and goals for Hypertension Research include the facilitation of experimental and clinical research activity in Asia, and fostering of collaboration between local and international researchers in the field. I would also like to inspire younger researchers in the hypertension area to submit their work for publication so that we can support them to grow and develop their research interests.

I encourage all researchers to submit and publish their papers in Hypertension Research. Together we can provide high-quality evidence that contributes to the fight against hypertension and the global pandemic of cardiovascular disease to improve the lives of patients worldwide. 